\title{
Effects of Activated Carbon on the Reduction of Benzo(a)pyrene in Artemisia argyi Extract
}

\author{
Sung-Hoon Lee* \\ Professor, Department of Pharmaceutical-Biomedical Engineering, College of Engineering, Cheongju University, Cheongju 28503, South Korea
}

\section{ABSTRACT}

Background and objective: Artemisia argyi has a long history as an effective treatment for various diseases. The detection of environmental pollutant benzo(a)pyrene, a known human carcinogen, in the leaves of Artemisia argyi is cause for concern. For medicinal plant extracts, both a reduction of benzo(a)pyrene as well as the maintained effectiveness of the compound are important. Therefore, in this study, we propose an optimized process for the addition and filtration of activated carbon to reduce benzo(a)pyrene and change the contents of the indicating substance(jaceosidine and eupatilin). Methods: Artemisia argyi EtOH extract containing 36 ppb of benzo(a)pyrene was added to 0.1, 0.5, 1.0, and 1.5\% (w/w) of activated carbon for 120 min and filtered using an activated carbon filter 1, 2, 3, and 5 times respectively. The content of benzo(a)pyrene and indicating substances in Artemisia argyi extract were then measured with high performance liquid chromatography (fluorescence and UV detectors).

Results: As the amounts of activated carbon powder and filtering cycles increased, the content of benzo(a)pyrene in the Artemisia argyi extract decreased. However, when activated carbon powder $1.5 \%$ was added to the extract, and when the activated carbon filter was filtered five times, the results were reduced by 15\% and 30 40\% respectively. The optimal extraction condition for reducing benzo(a)pyrene was adding 1.5\% of activated carbon powder. This resulted in reducing benzo(a)pyrene by $83 \%$ and indicating substances by about $4 \%$.

Conclusions: Here we present a process for reducing benzo(a)pyrene in Artemisia argyi extract using activated carbon to reduce toxicity and minimize the loss of active ingredients. This approach has potential application within a manufacturing process of various medicinal plant extracts.

Keywords: extraction, HPLC, medicinal plants, process optimization

\section{Introduction}

Artemisia argyi, commonly known as mugwort, is a herbaceous perennial plant in the Asteracea family, and has strong vitality, being distributed in most of the regions in the world. Artemisia argyi is observed mainly in Northern temperate regions, in particular, Asia, Europe and North America. As it has a unique taste and scent, it has been used in various ways in the form of hardy plants or as an additive to foods and medical supplies. Artemisia argyi in Eastern medicine is known to have the efficacy of hemo- stasis, painkiller, diuresis, hypertension, fever alleviation and anti-inflammation and has been used as a blood supplement and sthenia and as a medicine for digestive diseases including chronic gastritis, abdominal pain, hematemesis and dyspepsia as well as women's diseases (Chen et al., 2013; Guo et al., 2013; Park et al., 2013; Ren et al., 2015; Yang et al., 2015). Recent studies also reported that Artemisia argyi has antibiotic, anticarcinogenic, antifungal and antioxidant effects, protects the liver and relieves diabetic symptoms (Bao et al., 2013; Nam et al., 2013; Wang et al., 2013; Zeng et al., 2014).

This study was supported by Academic Research Support Program of the Research Institute of Industrial Sciences of Cheongju University. Received: August 7, 2020, Revised: September 15, 2020, Accepted: September 21, 2020

First author: Sung-Hoon Lee, pharming.cju@gmail.com, (1) https://orcid.org/0000-0003-0885-6252

*Corresponding author: Sung-Hoon Lee, pharming.cju@gmail.com, (10) https://orcid.org/0000-0003-0885-6252 
The main substance of the leaves and stems of Artemisia argyi is cineol and it also contains a large amount of adenine, choline, tricosanol, hentriacontane, $\alpha$-thujone, amylase, stearic acid, fat, calcium, phosphorus, carbohydrates, proteins, iron and vitamin A, having both pharmacological and aesthetic effects (Park et al., 2015). Artemisia argyi can be used in various forms, and its extract is orally administered or is externally used as a moxa treatment. Since the substances of Artemisia argyi differ depending on its species, analysis methods and solvents used to extract, many analysis methods have been reported (Lee et al., 1996; Oh et al. 1997). In Korea, Dong-A ST developed a drug made of the $95 \%$ ethanol extract of Artemisia argyi to improve gastric mucosal lesions and to prevent diseases in the gastric mucosa caused by the administration of acute and chronic gastritis drugs and non-steroidal anti-inflammatory drugs (NSAID) in 2005 and has sold the drug since then. In particular, N-Nitrosodimethylamine (NDMA), a carcinogenic compound, was excessively detected in drugs that contain ranitidine used as an antiulcer drug and the drugs were withdrawn from the market in 2019. Since then, the market for the medical supplies that contain Artemisia argyi has significantly grown to about 90 billion won as of 2019 (Oh et al., 2001, Seol et al., 2004).

Benzo(a)pyrene is one of the organic matters with PAHs (Polycyclic aromatic hydrocarbons) and carbon and hydrogen atoms condensed with at least 2 aromatic rings (Fig. 1), and is known to have various toxicities when it is exposed to the endocrine system of the body as a kind of environmental pollutant (International Agency for Research on Cancer[IARC], 2006). PAHs are generated as a heat degradation product when lipids, carbohydrates and proteins are exposed to high temperature and are known to be carcinogenic and cause mutations. For this reason, PAHs are classified as Group 1 (carcinogenic to humans) and are controlled by the IARC(Mastrel and Callean, 2000; IARC, 2006). Humans are exposed to benzo(a)pyrene and PAHs that contaminate air, soil and water through automobile exhausts, incomplete combustion of fossil fuel, cigarette smoke, fat and oils heated while cooking and meats and fish smoked or heated with charcoal (Mumtaz and George, 1995).

Benzo(a)pyrene was detected in medicinal plants categorized as a medicinal product such as black ginseng (34

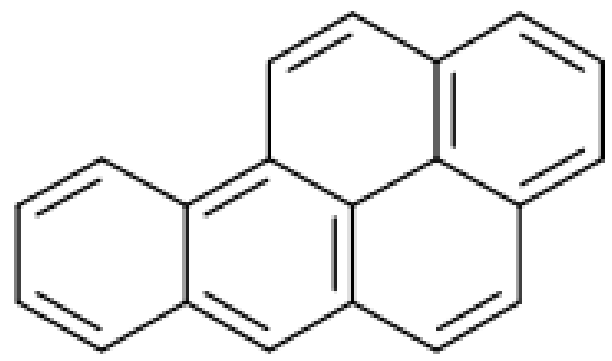

Fig. 1. Chemical structure of benzo(a)pyrene

$\mu \mathrm{g} / \mathrm{kg}$ ) in 2007 and Rehmannia glutinosa in 2008, which was raised as a social issue in Korea. Activated carbon is a form of carbon made from palm shells or coal, and is amorphous carbon with fine pores created in the process of activation by burning the materials at high temperature (Cho et al. 2012). The raw materials of activated carbon can be classified into plants, animals, minerals and industrial wastes, and in the case of minerals, coal types are used. In general, plants are used to produce activated carbon powder, and coals, charcoal and palm shells are used to produce granular activated carbon. The adsorption mechanism of activated carbon for removing pollutants is a phenomenon in which certain substances in liquids or gases are concentrated on the solid-liquid, gas-liquid and liquid-liquid interface. Adsorption can be divided according to shape into physical adsorption and chemical adsorption. The force involved in physical adsorption is the van der Waals force while chemical bonds such as covalent bond or ionic bond are involved in chemical adsorption. In the case of physical adsorption, when the gravitation force of molecules between target substance and adsorbent is greater than that between target substance and solvent, the target substance is adsorbed on the surface of the adsorbent (Franz et al., 2000).

As benzo(a)pyrene was detected in herbal medicinal products, the Herbal Medicinal Products Division of the National Institute of Food and Drug Safety Evaluation under the Ministry of Food and Drug Safety ordered measures to reduce benzo(a)pyrene in raw medicinal herbs and finished medicinal products, and established criteria for the margin of exposure (MOE) of benzo(a)pyrene as the daily maximum dose (Ministry of Food and Drug Safety[MFDS], 2015). As shown in Table 1, the MOE of benzo(a)pyrene as the daily maximum dose is targeted to be $10^{6}$ or higher. 
Table 1. MOE specification of benzo(a)pyrene in herbal extracts and products

\begin{tabular}{cc}
\hline MOE & Means \\
\hline$<10,000$ & Possible concern \\
$>10,000$ & Low concern \\
$>100,000$ & Negligible concern with action minimizing future exposure \\
$>1,000,000$ & Negligible concern \\
\hline MOE $=$ & BMDL $_{10}(0.1 \mathrm{mg} / \mathrm{kg} /$ day $) \times 10^{6}$ \\
\hline
\end{tabular}

No method has been reported to reduce benzo(a)pyrene in Artemisia argyi extract. When using the existing extraction methods for foods, the indicating substances of Artemisia argyi extract can be transformed by heat and their content can be reduced, affecting its efficacy, and thus it is necessary to develop measures to address this problem. Against this backdrop, this study aimed to develop a method of reducing benzo(a)pyrene that exists in Artemisia argyi extract using an activated carbon filter and powder.

\section{Research Methods}

\section{Reagents and solvents}

Artemisia argyi (collected from Cheongsong, Gyeongbuk) used as a crude herbal material was purchased from Gyeongdong Market and the standard of benzo(a)pyrene, an analysis target, was purchased from Sigma-Aldrich (MO, USA). Acetonitrile, methanol, n-hexane and ethyl acetate were purchased as an analysis solvent from Burdick \& Jackson (NJ, USA), and acetic acid glacial and absolute ethanol were purchased from Daejung (Gyeonggi, Korea). As an analysis reagent, ammonium acetate was purchased from Junsei (Tokyo, Japan), and 3-methylcholanthrene was purchased from Sigma-Aldrich (MO, USA) and was used as an internal standard for analyzing benzo(a)pyrene. The standards of eupatilin and jaceosidine - indicating substances of Artemisia argyi - were purchased from PhytoLab $\mathrm{GmbH} \&$ co. (Vestenbergsgreuth, Germany). The activated carbon powder used to reduce benzo(a)pyrene in this study was purchased from OMT Korea (Gyeonggi, Korea) and an activated carbon filter (RFC-20BB) was purchased from PENTEK (PA, USA). The florisil cartridge used to analyze benzo(a)pyrene was purchased from Waters (MA, USA).

\section{Analysis equipment}

High performance liquid chromatography (HPLC) analysis was conducted using Agilent 1260 Infinity series systems (Agilent, CA, USA). Agilent G1321C 1260 FLD (Agilent, CA, USA), a fluorescence detector, was used to analyze benzo(a)pyrene, and Agilent G114F 1260 VWD (Agilent, CA, USA), a UV detector, was used to analyze the indicating substances of Artemisia argyi. As an auto-sampler for analyzing samples, Agilent G1329B 1260 ALS (Agilent, CA, USA) was used. Agilent G1311C 1260 Quat Pump VL (Agilent, CA, USA) was used as a pump, and Agilent G1316A 1260 TCC (Agilent, CA, USA) as a column oven. Unison US-C 18 (Imtakt Corp, Japan) was used as a column for analyzing benzo(a)pyrene, and Inertsil ODS-2 $\mathrm{C}_{18}$ (GL Scieneces, Japan) was used for analyzing the indicating substances of Artemisia argyi. As a program for processing data, Agilent OpenLaB CDS [EZChrom Edition] A.04.06, A.04.07 version (Agilent Technologies, CA, USA) was used.

\section{Benzo(a)pyrene analysis method}

The standard of benzo(a)pyrene was dissolved with acetonitrile at a concentration of $50 \mu \mathrm{g} / \mathrm{mL}$ to make a standard stock solution. After that, $1 \mathrm{~mL}$ of the standard stock solution was added with acetonitrile to a total volume of 100 $\mathrm{mL}$ (Standard Solution-1 $(0.5 \mu \mathrm{g} / \mathrm{mL})$ ) and $10 \mathrm{~mL}$ of Standard Solution-1 was added with acetonitrile to a total volume of $100 \mathrm{~mL}$ (Standard Solution-2 (50 ng/mL)). 3-methylcholanthrene, an internal standard, was dissolved with acetonitrile at a concentration of $50 \mu \mathrm{g} / \mathrm{mL}$ to make an original internal standard solution. After that, $1 \mathrm{~mL}$ of 
the original internal standard solution was added with acetonitrile to a total volume of $100 \mathrm{~mL}$ (Internal Standard Solution-1 $(0.5 \mu \mathrm{g} / \mathrm{mL}))$ and $20 \mathrm{~mL}$ of Internal Standard Solution-1 was added with acetonitrile to a total volume of $100 \mathrm{~mL}$ (Internal Standard Solution-2 (100 ng/mL). Acetonitrile was used as a blank. After that, 0.4, 2, 4, 6 and $8 \mathrm{~mL}$ of Standard Solution-1 were accurately measured, and were mixed with $5 \mathrm{~mL}$ of Internal Standard Solution-2 and with acetonitrile to a total volume of 20 $\mathrm{mL}$ respectively, and each standard solution was ensured to have $1,5,10,15$ and $20 \mathrm{ng}$ of benzo(a)pyrene and 25 ng of the internal standard respectively. Their calibration curve was obtained. Artemisia argyi used as a crude herbal material and its extract $5 \mathrm{~g}$ were mixed homogeneously and $0.5 \mathrm{~g}$ was accurately measured and was mixed with $10 \mathrm{~mL}$ of anhydrous ethanol. Ultrasonic extraction was performed for 10 minutes, and $100 \mathrm{~mL}$ of water, $100 \mathrm{~mL}$ of hexane and $0.25 \mathrm{~mL}$ of Internal Standard Solution-2 were added to the extract, and shaking extraction was performed for 20 minutes. After that, the hexane layer was separated by centrifuging the mixed solution at 3,000 rpm for 10 minutes and the same procedure was repeated twice. The collected hexane layer was combined and transferred to a separating funnel and $50 \mathrm{~mL}$ of water was added to remove the remaining liquid layer. Using a filter containing sodium sulfate anhydrous, the hexane layer was dehydrated and filtered. After that, the layer was decompressed at about 700 bar in a water bath at $40^{\circ} \mathrm{C}$ to concentrate it until about $2 \mathrm{~mL}$ was left. First, the concentrated solution was added to a florisil cartridge that slowly activated 10 $\mathrm{ml}$ of dichloromethane and $20 \mathrm{ml}$ of hexane in order, and $30 \mathrm{~mL}$ of the hexane/dichloromethane solution (3:1) was eluted at the speed of 2-3 drops per second. The eluted solution was purged using nitrogen gas and the residue was melted with $1 \mathrm{~mL}$ of acetonitrile. After that, the liquid was filtered with a $0.45 \mu \mathrm{m}$ membrane filter to test. The standard solution and the tested liquid were analyzed under the analysis conditions shown in Table 2, and the analysis results of the tested liquid were substituted to the calibration curve obtained from the standard solution to calculate the content of benzo(a)pyrene.

\section{Method of analyzing indicating substances of Artemisia argyi extract}

The standard stock solution of eupatilin was prepared by dissolving $5 \mathrm{mg}$ of the standard of eupatilin in $50 \mathrm{~mL}$ of anhydrous ethanol, and the standard stock solution of jaceosidine was prepared by dissolving $5 \mathrm{mg}$ of the standard of jaceosidine in $100 \mathrm{~mL}$ of anhydrous ethanol. After that, $10 \mathrm{~mL}$ of each of the standard stock solutions was mixed with $100 \mathrm{~mL}$ of anhydrous ethanol to make a standard solution. About $60 \mathrm{mg}$ of the Artemisia argyi ethanol soft extract was added to and was completely dissolved in $80 \mathrm{~mL}$ of anhydrous ethanol and $100 \mathrm{~mL}$ was measured to use as a test liquid. The standard solution and the test liquid were analyzed under the analysis conditions shown in Table 2 to calculate the content of eupatilin and jaceosidine. The mobile phase used in this process was produced by dissolving $10 \mathrm{~g}$ of ammonium acetate in $500 \mathrm{~mL}$ of distilled water and adding $5 \mathrm{~mL}$ of acetic acid and distilled water to a total volume of $1000 \mathrm{~mL}$, which was used as an acetic acid-ammonium acetate buffer. The buffer and acetonitrile were mixed in the ratio of 65 to 35 .

Table 2. Analytical conditions of HPLC system with fluorescence and UV detector

\begin{tabular}{|c|c|c|}
\hline & Benzo(a)pyrene & Artemisia argyi extracts \\
\hline Wavelength & $\begin{array}{l}\text { Excitation: } 294 \mathrm{~nm} \\
\text { Fluorescence: } 404 \mathrm{~nm}\end{array}$ & UV: $350 \mathrm{~nm}$ \\
\hline Mobile phase & $72 \%$ Acetonitrile solution & $\begin{array}{c}\text { Acetic acid glacial-Ammonium acetic acid } \\
\text { Buffer }: \text { Acetonitrile }=65: 35\end{array}$ \\
\hline Flow rate & $1.0 \mathrm{ml} / \mathrm{min}$ & $1.0 \mathrm{ml} / \mathrm{min}$ \\
\hline Injection volume & $10 \mu \mathrm{l}$ & $10 \mu \mathrm{l}$ \\
\hline Column Temp. & $37^{\circ} \mathrm{C}$ & $25^{\circ} \mathrm{C}$ \\
\hline
\end{tabular}




\section{Comparison of benzo(a)pyrene reduction effects depending on the method of washing and drying crude herbal material}

To examine changes in the content of benzo(a)pyrene depending on the part of the crude herbal material used in this study and the application of washing, the crude herbal material was divided into leaves, stems and roots, and were cut into 1-2 $\mathrm{cm}$ pieces and the content of benzo(a)pyrene was measured. To identify the effect of washing the crude herbal material on the content of benzo(a)pyrene, the pieces of leaves were washed in the running water three times and the content of benzo(a)pyrene was measured. To examine changes in the content of benzo(a)pyrene depending on the method and temperature of drying the crude herbal material, the material was cut into small pieces of $5 \mathrm{~mm}$ or less and was dried in a shaded and sunny space for 3 days respectively. After that, it was dried at $60{ }^{\circ} \mathrm{C}$ in a drying oven for 10 hours. In addition, to examine changes in the content of benzo(a)pyrene depending on the temperature of drying, the crude herbal material of the same size was dried in a drying oven at 60,100 and $150{ }^{\circ} \mathrm{C}$ for 10 hours respectively and the content of benzo(a)pyrene was measured.

\section{Comparison of benzo(a)pyrene reduction effects depending on the application of activated carbon in the process of extraction}

To produce Artemisia argyi extract, $1 \mathrm{~kg}$ of the crude herbal material was mixed with $8 \mathrm{~L}$ of $95 \%$ ethanol, and the first extraction was performed at room temperature using a mechanical stirrer for 20 hours. After that, $8 \mathrm{~L}$ of $95 \%$ ethanol was added to it, and the second extraction was performed for 4 hours. After completing extraction, the extract was filtered using a 10 um filter and was concentrated at $55 \pm 5{ }^{\circ} \mathrm{C}$ and $-0.06 \mathrm{Mpa}$ or higher for 6 hours, and was concentrated again under the same conditions for 4 hours until the final mass of the extract becomes $50 \mathrm{~g}$ to obtain the standard extract. To identify the effect of adding activated carbon on the reduction of benzo(a)pyrene, as shown in Fig. 2, the extract after the second extraction was added with activated carbon powder (average particle size, 200 mesh) of $0.1,0.5,1.0$ and $1.5 \%$ of the mass of the crude herbal material, was stirred at $40{ }^{\circ} \mathrm{C}$ for 2 hours and was filtered using a 10 um filter to remove activated carbon. In addition, the extract was filtered using an industrial-grade activated carbon filter ( $508 \times 114 \mathrm{~mm}, 25 \mu \mathrm{m}$ ) $1,2,3$ and 5 times respectively to analyze the content of benzo(a)pyrene to compare the reduction effect.

\section{Results and Discussion}

\section{Benzo(a)pyrene reduction effects depending on the method of washing and drying crude herbal material}

The content of benzo(a)pyrene in each part of the crude herbal material was analyzed, and as shown in Table 3, $2.25( \pm 0.40)-2.51( \pm 0.13) \%$ was found in stems and roots,

Table 3. Changes of the content of benzo(a)pyrene in medicinal plants by water washing

\begin{tabular}{ccc}
\hline Part & Washing & $\begin{array}{c}\text { Mean Benzo(a)pyrene content } \\
(\mathrm{ng} / \mathrm{g} \pm \mathrm{SD})\end{array}$ \\
\hline Leaf & Washed & $5.77 \pm 0.24$ \\
& Non-washed & $7.12 \pm 0.25$ \\
Stem & - & $2.25 \pm 0.40$ \\
Root & - & $2.51 \pm 0.13$ \\
\hline
\end{tabular}

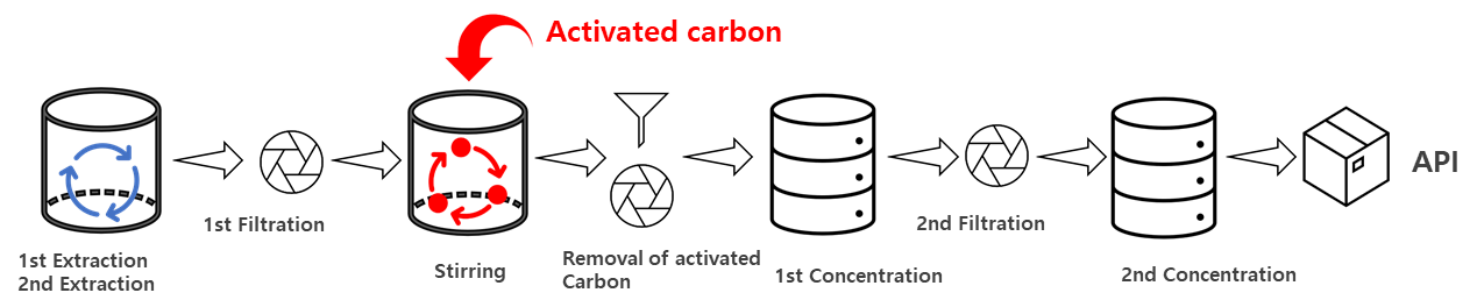

Fig. 2. Scheme of extraction method for reducing benzo(a)pyrene. 
Table 4. Changes of the content of benzo(a)pyrene in medicinal plants by drying method and temperature

\begin{tabular}{ccc}
\hline Drying method & Drying condition & $\begin{array}{c}\text { Mean Benzo(a)pyrene } \\
\text { content }(\mathrm{ng} / \mathrm{g} \pm \mathrm{SD})\end{array}$ \\
\hline \multirow{2}{*}{ Natural drying } & Shady place $/ 3$ days & $3.62 \pm 0.06$ \\
& Sunny place $/ 3$ days & $3.85 \pm 0.52$ \\
\multirow{5}{*}{ Drying oven } & $60^{\circ} \mathrm{C} / 10$ hours & $4.51 \pm 0.36$ \\
& $100^{\circ} \mathrm{C} / 10$ hours & $5.26 \pm 0.33$ \\
& $150^{\circ} \mathrm{C} / 10$ hours & $5.14 \pm 0.09$ \\
\hline
\end{tabular}

4.61 4.87\% lower than the leaves that were not cleaned. The content in the leaves cleaned with water was reduced by $1.35 \%$ from the content before washing. The content of benzo(a)pyrene was high in the leaves that had a relatively large surface area compared to stems or roots, which can be attributed to differences in the content due to the adsorption of benzo(a)pyrene in the air. The methods of natural drying and hot-air drying were compared as shown in Table 4, the crude herbal material dried naturally showed a decrease of $0.7 \sim 1.6 \%$ on average in the content of benzo(a)pyrene. The methods of drying in a shaded and sunny space were also compared, but no significant difference $(0.2 \%)$ was observed. In the case of hot-air drying, the higher the temperature, the higher the content of benzo(a)pyrene, but there was no significant difference at the temperature of over $100{ }^{\circ} \mathrm{C}$. When the material was dried at over $100{ }^{\circ} \mathrm{C}$, the unique scent of Artemisia argyi disappeared and the color of the dried material turned to dark brown, showing changes in its properties.

\section{Benzo(a)pyrene reduction effects depending on the extraction method of crude herbal material}

In the process of producing the Artemisia argyi ethanol extract, the extract added with activated carbon power was stirred and was filtered with an activated carbon filter after extraction to identify the effects of activated carbon on the reduction of benzo(a)pyrene. Differences in the effects of activated carbon powder were also tested by adding activated carbon powder of $0.1,0.5,1.0$ and $1.5 \%$ of the mass of the crude herbal material and mixing it for 2 hours. As shown in Fig. 3, when activated carbon powder of $0.1 \sim 0.5 \%$ was added, there was no significant difference
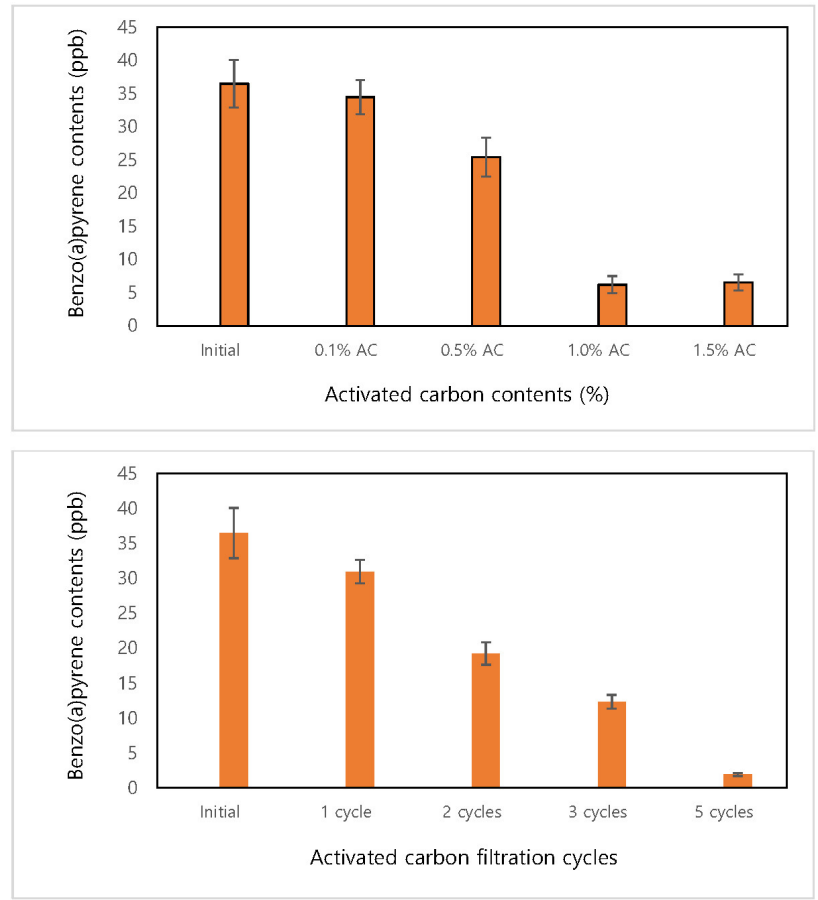

Fig. 3. Reduction effect of benzo(a)pyrene according to activated carbon filter and powder

in benzo(a)pyrene compared to the existing process, but when activated carbon powder of $1.0 \%$ and $1.5 \%$ was added, the content of benzo(a)pyrene was reduced by about $94 \%$ compared to the existing process. There was no significant difference between $1.0 \%$ and $1.5 \%$, indicating that increasing the amount of activated carbon powder further is not effective. In addition, the effect of using an activated carbon filter depending on the number of filtrations was examined, and it was found that the higher the number of filtration, the lower the content of benzo(a)pyrene proportionally. Compared to activated carbon powder, the benzo(a)pyrene reduction effect of filtering the extract five times using an activated carbon filter was higher by over $5 \%$. When filtering five times repeatedly showed a decrease in the content of benzo(a)pyrene by about $98 \%$ compared to the existing process, and thus filtration was not repeated more than five times in this study.

Changes in the content of eupatilin and jaceosidine, the active indicating substances of Artemisia argyi, using activated carbon powder and filter of which reduction effect was proved, were examined. The loss of the indicating substances when the extract was mixed and filtered with acti- 


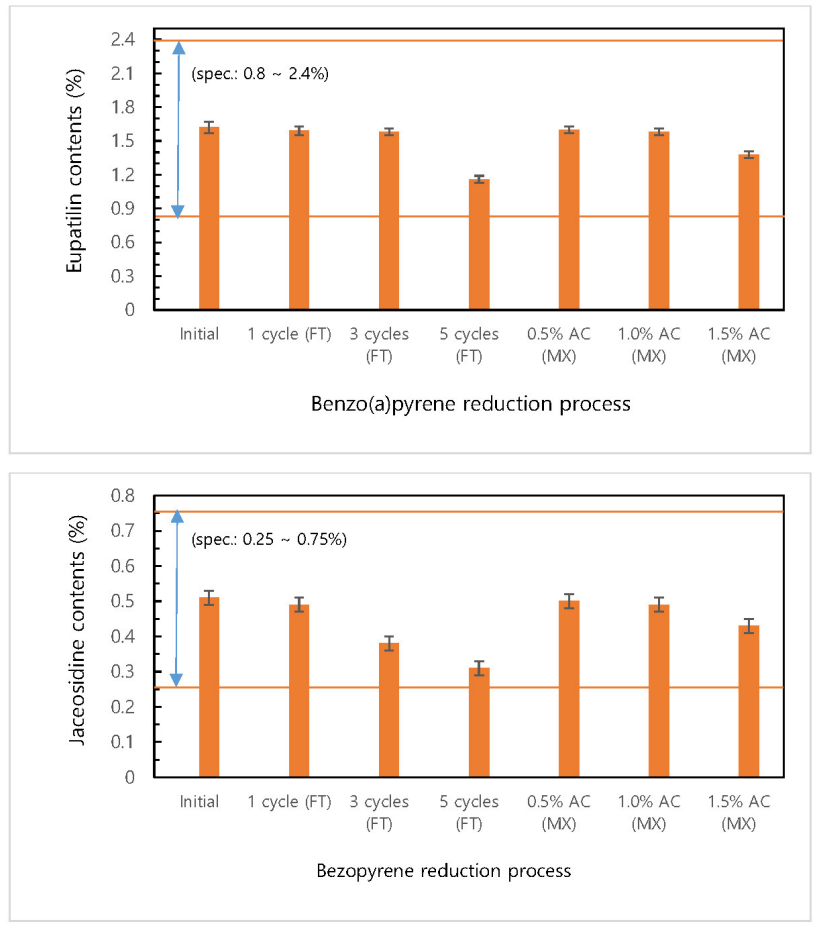

Fig. 4. Changes in the content of indicating substances according to the benzo(a)pyrene reduction process

vated carbon to remove benzo(a)pyrene was tested using HPLC analysis. As shown in Fig. 4, there was no significant change in the content of benzo(a)pyrene when the amount of activated carbon powder was increased, but when the extract was repeatedly filtered using an activated carbon filter five times, there was a loss of about $30 \%$ in the content of eupatilin and a loss of about $40 \%$ in the content of jaceosidine. When activated carbon power of $1.0 \%$ was added, both eupatilin and jaceosidine showed a decrease by less than $4 \%$. However, when activated carbon powder of $1.5 \%$ was added, both eupatilin and jaceosidine showed a decrease of about $15 \%$, showing a similar result to when using an activated carbon filter, which can be attributed to the adsorption of indicating substances caused by the excessive input of activated carbon. Since Artemisia argyi extract is used as a raw material for medical supplies, the benzo(a)pyrene reduction efficiency is important, but the loss of indicating substances that have medicinal effects is also an important indicator from the aspect of functions as a medical supply. Therefore, the reduction method that can satisfy both seems to be suitable.

\section{Conclusion}

In this study, it was found that benzo(a)pyrene is mostly distributed on the leaves of medicinal plants, which seems to be caused by the adsorption of benzo(a)pyrene in the air. In addition, the method that can satisfy both the reduction of benzo(a)pyrene and the maintenance of indicating substances in medicinal plants was found to be additionally mixing activated carbon powder of $1.0 \%$ and concentrating. It was also found that the excessive input of activated carbon powder that can reduce the content of benzo(a)pyrene through adsorption can reduce the content of indicating substances. Therefore, it will be necessary to consider as an important indicator the reduction of benzo(a)pyrene as well as changes in the content of indicating substances in producing the extract of medicinal plants used in functional foods and medical supplies, contributing to the development of production methods that are advantageous both from the aspect of efficacy and harmfulness.

\section{References}

Bao, X., H. Yuan, C. Wang, J. Liu, and M. Lan. 2013. Antitumor and immunomodulatory activities of a polysaccharide from Artemisia argyi. Carbohydr. Polym. 98(1): 1236-1243. https://doi.org/ 10.1016/j.carbpol.201 3.07.018

Chen, R., M. Chen, J. Xiong, Z. Chi, B. Zhang, N. Tian, Z. Xu, T. Zhang, W. Li, W. Zhang, X. Rong, Z. Wang, G. Sun, B. Ge, G. Yu, and N. Song. 2013. Curative effect of heat-sensitive moxibustion on chronic persistent asthma: a multicenter randomized controlled trial. J. Tradit. Chin. Med. 33(5): 584-591. https://doi.org/10.10 16/s0254-6272(14)60025-x

Cho, Y.H., K.Y. Song, M.K. Baek, J.W. Lee, and G.W. Lee. 2012. Study on extraction condition and analysis methods of benzopyrene in black ginseng. Yakhak Hoeji. 56(3):145-151.

Franz M., H.A. Arafat, and N.G. Pinto. 2000. Effect of chemical surface heterogeneity on the adsorption mechanism of dissolved aromatics on activated carbon. Carbon 38(13): 1807-1819. https://doi.org/10.1016/S000 8-6223(00)00012-9 
Guo, J., L.P. Wang, C.Z. Liu, J. Zhang, G.L. Wang, J.H. Yi, and J.L. Cheng. 2013. Efficacy of acupuncture for primary insomnia: a randomized controlled clinical trial. Evid. Based Complement Alternat. Med. 2013(163850). https://doi.org/10.1155/2013/163850

International Agency for Research on Cancer. 2006. IARC Monographs on the evaluation of carcinogenic risks to humans "Some non-heterocyclic polycyclic aromatic hydrocarbons and some related exposures". 92. WHO IARC.

Lee, E.B., S.A. Cheon, E.S. Lee, K.K. Kim, S.T. Ko, K.J. $\mathrm{Yu}$, and M.H. Sohn. 1996. General pharmacology of artemisia extract powder, DA-9601. J. Appl. Pharmacol. 4(2):174-183.

Mastral A.M., and M.S. Callean. 2000. A review on polycyclic aromatic hydrocarbon(PAH) emissions from energy generation. Environ. Sci. Technol. 34(15):3051-3057. https://doi.org/10.1021/es001028d

Ministry of Food and Drug Safety. 2015. Order to reduce the detection of benzopyrene in natural medicines. Herbal Medicine Policy Division-4688

Mumtaz M., and J.D. George. 1995. Toxicological profile for polycyclic aromatic hydrocarbons(PAHs). Georgia, US: Agency for Toxic Substances and Disease Registry.

Nam Y., M. Choi, H. Hwang, M.G. Lee, B.M. Kwon, W.H. Lee, and K. Suk. 2013. Natural flavone jaceosidin is a neuroinflammation inhibitor. Phytother. Res. 27(3):404-411. https://doi.org/10.1002/ptr.4737

Oh, T.Y., B.O. Ahn, J.I. Ko, B.K. Ryu, M.W. Son, S.H. Kim, and E.B. Lee. 1997. Studies on protective effect of da-9601, an artemisiae herbal extract, against ethanol-induced gastric mucosal damage and its mechanism. J. Appl. Pharmacol. 5(2):202-210.

Oh T.Y., J.S. Lee, B.O. Ahn, H. Cho, W.B. Kim, Y.J. Kim, Y.J. Surh, S.W. Cho, K.M. Lee, and K.B. Hahm. 2001. Oxidative stress is more important than acid in the pathogenesis of reflux oesophagitis in rats. Gut
49(3):364-371. https://doi.org/10.1136/gut.49.3.364.

Park J.Y., D. Lee, H.J. Jang, D.S. Jang, H.C. Kwon, K.H. Kim, S.N. Kim, G.S. Hwang, K.S. Kang, and D.W. Eom. 2015. Protective effect of Artemisia asiatica extract and its active compound eupatilin against cisplatin-induced renal damage. Evid. Based Complement Alternat. Med. 2015(483980). https://doi.org/10.1155/2015/483980

Park J.W., B.H. Lee, and H. Lee. 2013. Moxibustion in the management of irritable bowel syndrome: systematic review and meta-analysis. BMC Complement. Altern. Med. 13:247. https://doi.org/10.1186/1472-6882-13-247

Ren, X., C. Yao, F. Wu, Z. Li, J. Xing, and H. Zhang. 2015. Effectiveness of moxibustion treatment in quality of life in patients with knee osteoarthritis: a randomized, double-blinded, placebo-controlled trial. Evid. Based Complement Alternat. Med. 2015(569523). https://doi.o rg/10.1155/2015/569523

Seol, S.Y., M.H. Kim, J.S. Rew, and M.G. Choi. 2004. A phase III clinical trial of Stillen(TM) for erosive gastritis. Korean J. Gastrointest. Endosc. 28(5):230-236.

Wang, S., J. Li, J. Sun, K.W. Zeng, J.R. Cui, Y. Jiang, and P.F. Tu. 2013. NO inhibitory guaianolide-derived terpenoids from Artemisia argyi. Fitoterapia 85:169-175. https://doi.org/10.1016/j.fitote.2012.12.005

Yang, J., S. Yu, L. Lao, M. Yang, J.P. Chen, X. Luo, Y. Wang, X. Chen, J. Li, L. Zhu, Q. Zheng, Y. Hu, $\mathrm{X}$. $\mathrm{Wu}$, and F. Linang. 2015. Use of moxibustion to treat primary dysmenorrhea at two interventional times: study protocol for a randomized controlled trial. Trials 16(1): 35. https://doi.org/10.1186/s13063-015-0552-1

Zeng, K.W., S. Wang, X. Dong, Y. Jiang, and P.F. Tu. 2014. Sesquiterpene dimer (DSF-52) from Artemisia argyi inhibits microglia-mediated neuroinflammation via suppression of NF-kappaB, JNK/p38 MAPKs and Jak2/Stat3 signaling pathways. Phytomedicine 21(3):298-306. https://doi.org/10.1016/j.phymed.2013.08.016 